Published in final edited form as:

Biochemistry. 2005 October 18; 44(41): 13425-13438.

\title{
Molecular dynamics simulations of salicylate effects on the micro- and mesoscopic properties of a dipalmitoylphosphatidylcholine
}

\section{bilayert}

\author{
Yuhua Song ${ }^{\ddagger}$, Victor Guallar§, and Nathan A. Baker ${ }^{*}$ \\ $\$$ Dept. of Biochemistry and Molecular Biophysics, Center for Computational Biology, Washington Univ. St. \\ Louis.E-mail: yhsong@ccb.wustl.edu
}

$\S$ Dept. of Biochemistry and Molecular Biophysics, Center for Computational Biology, Washington Univ. St. Louis.E-mail:guallarv@biochem.wustl.edu

\begin{abstract}
Salicylate, an amphiphilic molecule and a popular member of non-steroidal antiinflammatory drug family, is known to affect hearing through reduction of the electromechanical coupling in the outer hair cells of the ear. This reduction of electromotility by salicylate has been widely studied but the molecular mechanism of the phenomenon is still unknown. In this study, we investigated one aspect of salicylate's action; namely, the perturbation of electrical and mechanical membrane properties by salicylate in the absence of cytoskeletal or membrane-bound motor proteins such as prestin. In particular, we simulated the interaction of salicylate with a dipalmitoylphosphatidylcholine (DPPC) bilayer via atomically-detailed molecular dynamics simulations to observe the effect of salicylate on the microscopic and mesoscopic properties of the bilayer. The results demonstrate that salicylate interacts with the bilayer by associating at the water-DPPC interface in a nearly perpendicular orientation and penetrating more deeply into the bilayer than either sodium or chloride. This association has several affects on the membrane properties. First, binding of salicylate to the membrane displaces chloride from the bilayer-water interface. Second, salicylate influences the electrostatic potential and dielectric properties of the bilayer, with significant changes at the waterlipid bilayer interface. Third, salicylate association results in structural changes including decreased head group area per lipid and increased lipid tail order. However, salicylate does not significantly alter the mechanical properties of the DPPC bilayer; bulk compressibility, area compressibility, and bending modulus were only perturbed by small, statistically-insignificant amounts, by the presence of salicylate. The observations from these simulations are in qualitative agreement with experimental data and support the conclusion that salicylate influences the electrical but not the mechanical properties of DPPC membranes.
\end{abstract}

\footnotetext{
${ }^{\dagger}$ This work was supported by NIH grant R01 GM069702 (NAB and YS) and an Alfred P. Sloan Research Fellowship (NAB).

* To whom correspondence should be addressed. Dept. of Biochemistry and Molecular Biophysics, Center for Computational Biology, Washington Univ. St. Louis. 700 S. Euclid Ave., Campus Box 8036, St. Louis, MO 63110. Phone: (314) 362-2040, Fax: (314) 362-0234, E-mail: baker@biochem.wustl.edu, Web: http://agave.wustl.edu/.

${ }^{1}$ Abbreviations: constant number-pressure-temperature (NpT), constant number-volume-temperature (NVT),

dipalmitoylphosphatidylcholine (DPPC), giant unilamellar vesicle (GUV), human embryonic kidney (HEK), molecular dynamics (MD), outer hair cell (OHC), particle-mesh Ewald (PME), quantum mechanical/molecular mechanical (QM/MM), root mean squared (RMS), salicylate (SAL), solvent-accessible surface area (SASA), stearoyl-oleoylphosphatidylcholine (SOPC)

Supporting Information Available

Additional data, including figures of the QM/MM setup, time courses for system area, volume, and potential energy, selected membrane dynamical properties, additional data for Figure 4 and Figure 5, and movies of the molecular dynamics simulations, have been provided as supporting information. This material is available free of charge via the Internet at http://pubs.acs.org/.
} 
Salicylate (2-hydroxybenzoic acid) is closely related to aspirin and is a member of the nonsteroidal anti-inflammatory family of drugs (1). Interestingly, salicylate has a number of hearing-related reversible side effects at high doses, including hearing loss, a decrease in spontaneous otoacoustic emissions, and tinnitus; all of which have been related, in part, to changes in the mechanical and electrical properties of the cochlear outer hair cell (OHC) (211). OHCs play an important role in the sensitivity of mammalian hearing for high-frequency sounds through a mechanism of electromechanical feedback (12). While the exact molecular mechanism of salicylate's effect on OHC electromechanical response is still unknown, experimental work has suggested that salicylate may interact with several components of the OHC, including the phospholipid bilayer (13-20) and membrane-bound proteins such as prestin (21-23). In this study, we have investigated one aspect of salicylate's effects on OHC electromechanical coupling: the perturbation of electrical and mechanical biomembrane properties by salicylate in the absence cytoskeletal components or membrane-bound motor proteins such as prestin. The goal of this study is to elucidate the atomic-scale influence of salicylate on the mesoscopic mechanical and electrical properties of a "model" dipalmitoylphosphatidylcholine (DPPC) membrane.

Molecular dynamics (MD) methods have proven to be a useful tool in studying several structural, energetic, and dynamic properties of lipid bilayers (24-28), as well as analyzing the interactions of various small molecules (29-38) and macromolecules (39-42) with biomembranes. This study leverages the tremendous effort that has gone into phospholipid force field development to use MD simulations to understand the effect of salicylate on the microscopic and mesoscopic properties of a model membrane.

\section{Materials and methods}

Molecular dynamics (MD) trajectories of the interaction of the salicylate with a DPPC bilayer were calculated with GROMACS 3.2.1 package (43). A total of six simulations were performed at varying salicylate and $\mathrm{NaCl}$ concentrations (see Table 1 and below). The following sections describe the methods used to set up and simulate each system.

\section{Force field parameters and starting structures}

The initial salicylate (SAL, 2-hydroxybenzoic acid, see Figure 1) structure was obtained from the HIC-Up database (44). This structure was transformed into a GROMACS united atom topology file using the PRODRG server (45). To obtain the all atom force field for the salicylate, non-polar hydrogens were added manually and the steric, torsional, and angle parameters were assigned based on the GROMACS force field in GROMACS 3.2.1 (43). The atomic charges of salicylate were calculated from quantum mechanical/molecular mechanical (QM/MM) calculations. In these QM/MM calculations, we started from the minimized DPPC/ $10 \mathrm{mM} \mathrm{SAL} / \mathrm{NaCl}$ system and selected a subsystem consisting of four salicylate molecules (in different conformations) and a solvation sphere of $1.6 \mathrm{~nm}$ around each of them (see Figure 1 in the Supporting Information). The system was further minimized using a QM/MM potential as implemented in the QSite program (46). The QM/MM methodology and protocol have been described extensively elsewhere $(47,48)$. The quantum region consisted of the four salicylate molecules and all water molecules within $0.3 \mathrm{~nm}$ of them, for a total of $135 \mathrm{QM}$ atoms. Geometry optimizations of salicylate were carried out using the B3LYP functional in combination with the 6-311G* basis set; the oxygen coordinates in the outermost $0.2 \mathrm{~nm}$ of solvation waters were held fixed. For the molecular mechanics potential energy function, QSite uses the OPLS-AA force field (49). The charges on the salicylate atoms were then obtained by fitting to the quantum electrostatic potential surface. The final charges used in the molecular dynamics simulation were obtained as the average of the four different salicylate units; these charges are listed in Table 2. While the differing local environments and conformations 
introduced small charge variations between the four salicylate molecules, adopting an (averaged) fixed charge parameter set for each salicylate was necessary for the long molecular dynamics runs. Hopefully, the advent of new efficient polarizable force fields (50) will obviate the need for fixed charges in future calculations.

The DPPC force field parameters used in this simulation were developed by Berger and Lindahl (51) and can be found in version 3.2.1 of the GROMACS software package (43). These DPPC parameters have been shown to successfully reproduce experimental quantities such as lipid volume (52) and order parameters (53). The initial structure of a 64-lipid DPPC bilayer composed obtained from the Tieleman group (54). A lipid bilayer composed of 256 DPPC lipids was generated by periodic replication of the 64-lipid Tieleman structure to give a system with lateral dimensions of roughly $10 \mathrm{~nm} \times 10 \mathrm{~nm}$. The choice of 256 DPPC lipids was a tradeoff between computational cost and the size of system required to obtain reasonable continuum properties based on the previous published studies (27).

All the simulations were performed in a roughly $10 \mathrm{~nm} \times 10 \mathrm{~nm} \times 10 \mathrm{~nm}$ periodic box, thus ensuring more than $2 \mathrm{~nm}$ of solvent between the lipid surface and box boundaries to reduce potential artifacts arising from periodicity. This box was filled with 14,422-15,384 SPC (55) water molecules (depending on system simulated) corresponding to a hydration level of 5660 waters per lipid. The SPC water model was chosen based on previous studies demonstrating its efficiency and relative accuracy for membrane simulations (26).

As mentioned above (see also Table 1), simulations were performed for six DPPC systems with different concentrations of salicylate and $\mathrm{NaCl}$. The first system (pureDPPC) contained a DPPC bilayer and water only; the second (DPPC/10mM SAL) contained 4 salicylates (corresponding to $10 \mathrm{mM}$ salicylate) and $4 \mathrm{Na}^{+}$(used to keep the system neutral); the third (DPPC/60mM SAL) contained 20 salicylates $\left(60 \mathrm{mM}\right.$ salicylates) and $20 \mathrm{Na}^{+}$; the fourth (DPPC/NaCl) contained $50 \mathrm{Na}^{+}$and $50 \mathrm{Cl}^{-}$(corresponding to $150 \mathrm{mM} \mathrm{NaCl}$ ); the fifth (DPPC/ $10 \mathrm{mM} \mathrm{SAL} / \mathrm{NaCl}$ ) contained 4 salicylates, $54 \mathrm{Na}^{+}$, and $50 \mathrm{Cl}^{-}$; and the sixth (DPPC/ $60 \mathrm{mMSAL} / \mathrm{NaCl}$ ) contained 20 salicylates, $70 \mathrm{Na}^{+}$, and $50 \mathrm{Cl}^{-}$. During the simulation system setup, the ions and salicylates were randomly distributed in the water with the constraint that the minimum solute-solute and solute-lipid distances were greater than $6 \AA$. This constraint was imposed to prevent artificial ion pairing in the initial stages of the simulation.

\section{Simulations}

All six simulations followed the same molecular dynamics protocol. First, steepest descent minimization and 20 ps of constant number-pressure-temperature (NpT) molecular dynamics were performed with mobile water molecules but with the lipid bilayer, salicylate, and ions restrained. This short water equilibration was carried out at $50 \mathrm{~K}$ with isotropic pressure scaling to $1 \mathrm{~atm}$ and time steps of $1 \mathrm{fs}$. Next, the system was warmed gradually via a series of $10 \mathrm{ps}$ constant number-volume-temperature (NVT) MD simulations at 50, 100, 150, 200, 250, and $323 \mathrm{~K}$ with SHAKE constraints and 2 fs time steps. Because of the slow relaxation of lipid bilayer systems $(27,56)$, an additional $5 \mathrm{~ns}$ NpT MD trajectory was performed to fully equilibrate the system. The production trajectory was obtained through $25 \mathrm{~ns}$ of NpT dynamics at $323 \mathrm{~K}$ and $1 \mathrm{~atm}$. The final temperature of $323 \mathrm{~K}$ was chosen to ensure simulation of the membrane in the liquid crystalline phase (57). The Parrinello-Rahman pressure coupling algorithm (58) was used to isotropically scale the pressure with a time constant of 2 ps while temperature was controlled by the Nosé-Hoover temperature coupling algorithm (59) with a time constant of $0.5 \mathrm{ps}$. Finally, SHAKE constraints were used on all hydrogen-heavy atom bonds to permit a dynamics time step of $2 \mathrm{fs}$. Electrostatic interactions were calculated with Particle-Mesh Ewald method (PME) (60), thus avoiding the artifacts caused by electrostatic cutoffs $(61,62)$. Both the direct space PME cutoff the Lennard-Jones cutoffs were set at 1.0 $\mathrm{nm}$. 
The simulations were performed on a variety of dual-processor shared memory machines, including 2-CPU Intel Pentium 4 (with Intel compilers), 2-CPU AMD Opteron (with Portland Group compilers), 2-CPU Apple G5 (with GNU compilers), and 4-CPU Intel Itanium2 (with Intel compilers). On average, $10 \mathrm{~ns}$ of simulation required roughly 2 weeks (wall clock) run time for the Itanium 2 and 4 weeks run time for the other platforms.

\section{Results and Discussion}

The following sections discuss the effects of salicylate on the mesoscopic (continuum) and microscopic (molecular and atomic) properties of the DPPC system. In addition to the quantities presented below, time courses of collective properties such as system area, volume, and potential energy are presented in Figures 2-4 of the Supporting Information. Given the relevance of salicylate's effect on $\mathrm{OHC}$ electromotility, we have focused the analysis of our MD simulations on membrane structural, electrical, and mechanical properties. Additional data on salicylate's effect on membrane dynamical properties is available in Supporting Information.

\section{Areas and volumes}

The head group or interfacial area per lipid molecule measures the average area occupied by each lipid in the bilayer (63). This quantity can be calculated from the projected area of the simulation box; i.e., the area spanned by the lateral dimensions of the system. The area per lipid head group is then the projected area divided by the number of lipid molecules. Using this definition, the average areas per lipid were calculated for the six systems and shown in Figure 2(a) with error bars obtained by the $5 \mathrm{~ns}$ block averaging scheme described above. The simulations produced an area per lipid for pure DPPC of $0.627 \pm 0.001 \mathrm{~nm}^{2}$, a value that is in reasonable agreement with the experimental result of $0.64 \mathrm{~nm}^{2}$ (64). Student $t$-test results showed that salicylate significantly decreased $(\mathrm{p}<0.05)$ the area per head group irrespective of $\mathrm{NaCl}$ concentration with significant dose-dependence in the presence of $\mathrm{NaCl}$.

The affect of salicylate on lipid volumes was also determined by calculating per-lipid volumes. The volume of the lipid bilayer was calculated by the multiplying the projected bilayer surface area by the average bilayer height as determined by the average lipid phosphorous position $(52,64)$. The per-lipid volume was then obtained by dividing the bilayer volume by the number of lipids. Using this definition, the average volumes per lipid were calculated for the six systems and shown in Figure 2(b) with error bars obtained by the $5 \mathrm{~ns}$ block averaging scheme described above. The simulations produced a volume per lipid for pure DPPC of $1.201 \pm 0.001 \mathrm{~nm}^{3}$, a value that is in good agreement with the experimental result of $1.23 \mathrm{~nm}^{3}(64)$. The presence of salicylate leads to a small reduction in per-lipid volume; however, this reduction is only significant in the absence of $\mathrm{NaCl}$. Additionally, this effect does not change systematically or significantly with salicylate concentration.

\section{Tail order}

The lipid tail order parameter is a standard quantity used to evaluate the structural ordering of acyl chains in a lipid bilayer. In experiments, deuterium order parameters for each $\mathrm{CH}_{2}$ $\left(\mathrm{CD}_{2}\right)$ group can be determined by nuclear magnetic resonance as $(65)$

$$
S_{\alpha \beta}=\frac{1}{2}\left(3\left\langle\cos \theta_{\alpha} \cos \theta_{\beta}\right\rangle-1\right) \alpha, \beta=x, y, z
$$

where $\theta_{\alpha}$ is the angle between the $i^{\text {th }}$ molecular axis and bilayer normal ( $z$ axis). The $z$-axis is the molecular axis per $\mathrm{CH}_{2}$ unit, $x$ and $y$ axes are assigned based on a right handed coordinate 
system. In MD simulations with united atom representations for the $\mathrm{CH}_{2} / \mathrm{CH}_{3}$ hydrocarbon groups, the deuterium order parameter can be determined from (65)

$$
-S_{C D}=\frac{2}{3} S_{x x}+\frac{1}{3} S_{y y}
$$

where $S_{x x}$ and $S_{y y}$ are the order parameters determined with equation (1) by the angle between the axis determined by the $(i-1)^{\text {th }}, i^{\text {th }},(i+1)^{\text {th }}$ carbon atom of the lipid tail and vector $x$ and $y$. These definitions provide interpretations for acyl chain order parameters in a bilayer: order parameters assume a maximum value of 1 when the relevant segments are uniformly aligned along the bilayer normal; the order parameters are at a minimum value of -0.5 when the segments are uniformly aligned in the bilayer plane.

The effect of salicylate on lipid tail order parameters are shown in Figure 3 for the cases with and without added $\mathrm{NaCl}$. The Student $t$-test (66) with 95\% confidence was used to compare whether the changes of tail order caused by salicylate were significant. For each carbon atom, the mean of the tail order was obtained by averaging the trajectory, and the deviation was calculated from $5 \mathrm{~ns}$ block averages of the data which was similar to the block averaging method presented by Flyvbjerg et al (67). The average simulated tail order parameters in the "plateau" region (carbon numbers 4-8; see Figure 3) of the acyl chain for the pure DPPC bilayer were measured at $-0.208 \pm 0.002$. This value is in good agreement with experimental measurements of $-0.20 \pm 0.02$ for the same system (53). Application of Student's $t$-test showed that salicylate significantly increased the tail order for systems with $150 \mathrm{mM} \mathrm{NaCl}$ and without $\mathrm{NaCl}$ $(p<0.05)$; however, the change of the tail order between different concentrations of salicylate was not significant (i.e., no dose-dependence). This increase in lipid tail order upon salicylate association is similar to the effect of cholesterol (34) but differs from the disordering effects (on some regions of the lipid acyl chain) of molecules such as halothane (68).

These changes, together with the area and volume values presented above, suggest that salicylate causes packing and ordering of the bilayer. The possible mechanisms for these phenomena are discussed in the context of salicylate interaction with the DPPC lipids in the following section.

\section{Interaction of salicylate with lipid bilayers}

Figure 4 illustrates the orientation of salicylate in the DPPC bilayer for the DPPC/60mMSAL/ $\mathrm{NaCl}$ system; similar plots for the other systems with salicylate are provided in Figure 5 of the Supporting Information. The most probable location of salicylate for all 4 SAL-containing simulations was at the water-bilayer interface, $2-3 \mathrm{~nm}$ away from the lipid bilayer center. The most probable orientation at the interface was nearly perpendicular with $\sim 30^{\circ}$ between the salicylate $\mathrm{Z} 1$ axis (see Figure 4a) and the membrane normal.

To further examine the interaction of the salicylate with the head group components of the lipid bilayer, radial distribution functions (RDF) between the salicylate hydrophilic group (defined below) and components of the lipid head group were calculated and are shown in Figure 5(a) for the DPPC/60mM-SAL/NaCl system. Using the atom naming scheme shown in Figure 1, the salicylate hydrophilic group was defined as the atoms $\mathrm{O} 1, \mathrm{O} 2, \mathrm{H} 2, \mathrm{O} 3$, and $\mathrm{C} 7$; hydrophobic group as the atoms C1, C2, C3, H3, C4, H4, C5, H5, C6, and H6. The plots in Figure 5(a) show the RDFs for the DPPC nitrogen, phosphate, phosphate oxygen and carbonyl oxygen around the center of salicylate hydrophilic group (defined above). These RDFs illustrate the nature of salicylate coordination by the DPPC head groups. A snapshot from the MD simulation depicting this coordination between salicylate and lipid is shown in Figure 5(b). Plots similar to Figure 5(a) for the other salicylate-containing simulations are provided in Figure 6 of the Supporting Information. 
The perpendicular insertion of salicylate into the DPPC bilayer and the tight coordination of salicylate by the DPPC head groups provide a mechanism to explain the reduction in surface area observed in the salicylate-containing simulations. In particular, the nearly-perpendicular insertion of the salicylate permits tight packing of lipids around the salicylate while coordination by the DPPC head groups draws neighboring lipids closer to the salicylate molecule. This mechanism predicts the ordering of lipids as demonstrated by the tail order parameters discussed above. A similar conclusion for reduction of lipid hydrophilic head group area by hydroxybenzoic acids was reached by Lin and co-workers (69) based on experimental observations of structural transitions in micelles. However, work by Zhou and Raphael on SOPC vesicles under tension demonstrates an increase in surface area upon application of salicylate (16). More differences between the current study and the work by Zhou and Raphael are discussed in the Mechanical properties section below.

\section{Effect of salicylate on ion distributions}

The binding of salicylate to the DPPC bilayer has a dramatic effect on co- and counter-ion distributions in the system. Figure 6 depicts the symmetrized distribution of $\mathrm{Na}^{+}$and $\mathrm{Cl}^{-}$ions along the bilayer normal direction. Figure 6(a) depicts a small, statistically-insignificant increase in $\mathrm{Na}^{+}$concentrations at the water-lipid interface for all salicylate concentrations. Figure 6(b) shows that the addition of $10 \mathrm{mM}$ salicylate to the DPPC system did not affect the distribution of $\mathrm{Cl}^{-}$in the system; however, the addition of $60 \mathrm{mM}$ salicylate significantly reduced $(\mathrm{p}<0.05)$ the local concentration of $\mathrm{Cl}^{-}$at the bilayer-water interface. This change in chloride distribution is likely the result of two factors: the competition of salicylate with $\mathrm{Cl}^{-}$ for coordination by the lipid head groups (70) and the electrostatic repulsion of $\mathrm{Cl}^{-}$by the salicylate negative charge. This "competition" between chloride and salicylate in the molecular dynamics simulations is indirectly supported by experimental studies which demonstrated a linkage between chloride concentration on the distribution of salicylate across the erythrocyte membranes (71). Competition between salicylate and chloride has also been directly observed in $\mathrm{OHC}$ studies by Oliver et al (21). While the effect in the $\mathrm{OHC}$ also involves interactions with the protein prestin, it indirectly supports our observation of salicylate competition with chloride in biomolecular binding.

Figure 6(e) and (f) shows the relative distribution of salicylate, $\mathrm{Na}^{+}$, and $\mathrm{Cl}^{-}$in the DPPC/ $10 \mathrm{mMSAL} / \mathrm{NaCl}$ and the DPPC/60mMSAL/NaCl systems. The results demonstrate that salicylate penetrates more deeply into the bilayer interior than either $\mathrm{Na}^{+}$or $\mathrm{Cl}^{-}$, especially for higher concentrations of salicylate $(60 \mathrm{mM})$. Given the amphiphilic nature of salicylate, this behavior is not surprising and is consistent with the DPPC coordination of salicylate discussed above. While the location of salicylate is predominantly influenced by its amphiphilic character, the deeper penetration of salicylate into the membrane bilayer may also be related to its larger size and potentially smaller (de-)solvation energy; as suggested by studies of Hofmeister-like ion effects in lipid bilayers (30).

\section{Electrostatic potential and dielectric properties}

The electrostatic potentials of the simulated systems were calculated along the bilayer normal (z). First, mean charge densities for $x y$-slices of the periodic box were obtained by averaging over the dynamics trajectories. Electrostatic potentials were then calculated from these average charge densities by solving Poisson's equation via an integral of the charge density along the $\mathrm{z}$ direction:

$$
\varphi(z)-\varphi\left(z_{\min }\right)=-\frac{1}{\varepsilon_{0}} \int_{z_{\min }}^{z} d z^{\prime} \int_{z_{\min }}^{z^{\prime \prime}} \rho\left(z^{\prime \prime}\right) d z^{\prime \prime}
$$

where $z_{\min }$ corresponds to the edge of the simulation box, $\varepsilon_{0}$ is the vacuum permeability, $\rho$ is the charge density along the $z$ direction. The electrostatic potentials of the simulated systems 
were obtained from the MD trajectory and symmetrized by averaging over the two leaflets of the membrane; the results are shown in Figure 7(a) and (b) with error bars obtained by the block averaging scheme described above. Student $t$-values were calculated for 3 different comparisons for the systems with (Figure 7d) and without (Figure 7c) added $\mathrm{NaCl}$ : the $10 \mathrm{mM}$ SAL systems with respect to the $0 \mathrm{mM}$ SAL systems, the $60 \mathrm{mM} \mathrm{SAL}$ systems with respect to the $0 \mathrm{mM}$ SAL systems, and the $60 \mathrm{mM} \mathrm{SAL}$ systems with respect to the $10 \mathrm{mM} \mathrm{SAL}$ systems; the resulting $t$-values are plotted in Figure 7(c) and (d). These results show that salicylate significantly increased $(\mathrm{p}<0.05)$ the electrostatic potential at the water-lipid interface for the simulations without $\mathrm{NaCl}$, but this effect did not show a significant dependence on salicylate concentration. For the simulated systems with $\mathrm{NaCl}$, only $60 \mathrm{mM}$ salicylate caused a significant increase $(\mathrm{p}<0.05)$ in the electrostatic potential at the water-lipid interface. This increase is in qualitative agreement with experiments demonstrating salicylate-induced changes in membrane potentials $(17,72)$ and surface charges $(15)$ for human embryonic kidney (HEK) cells as well as various types of neurons (73-76).

Changes in electrostatic potential can occur for a number of reasons, including variations in local concentrations of counterions (as demonstrated above) or through alteration of the dielectric response properties of the system. The dielectric response of the system is related to the dipole fluctuations through linear response theory (77). Previous work by Lin et al (78) as well as more recent theoretical models by Ballenegger and Hansen (77) have shown that the dielectric influence of a membrane-like inclusion in water extends several hydration layers beyond the water-membrane interface. Therefore, we calculated dielectric coefficients for the entire system as a function of position along the membrane normal. In particular, local dipole moment fluctuation tensors $\Gamma$ were calculated according to

$$
\Gamma_{\alpha \beta}=\left\langle M_{\alpha} M_{\beta}\right\rangle-\left\langle M_{\alpha}\right\rangle\left\langle M_{\beta}\right\rangle \alpha, \beta=x, y, z
$$

via decimation of the simulation box into a series of $2.5 \AA$ cubic cells. Dipole moment fluctuation tensors were calculated for each cell and these results were integrated over the $x$ and $y$ directions to provide values along the membrane normal. Mean $\Gamma$ values were obtained by averaging over the MD trajectory; variances were calculated using the $5 \mathrm{~ns}$ block averaging method described above. The results of this analysis are shown in Figure 8 and indicate that salicylate significantly affected $(\mathrm{p}<0.05)$ dipole moment fluctuations in the system in a doseindependent manner. In particular, salicylate increased dipole moment fluctuations in the solvent region near the water-lipid interface $(\mathrm{z}=2-3 \mathrm{~nm})$ and decreased dipole moment fluctuation in the lipid bilayer region near the water-lipid interface $(z=3-4 \mathrm{~nm})$.

From these analyses, we can conclude that salicylate influences the electrical properties of the membrane system in at least two ways. First, it shifts the distribution of ions at the membrane interface and thereby changes the charge density near the water-bilayer interface. Second, salicylate interacts with the lipid bilayer, bound waters, and ions at the water-lipid interface and thereby reduces the dielectric response of portions of the lipid membrane and increases the response of interfacial water. Both of these changes are likely to be important in the perturbation of cell electrical behavior by salicylate.

\section{Mechanical properties}

Cell membrane mechanical properties are known to play important roles in cellular function through their permeability and deformability. For example, previous studies have shown that the elastic properties of a lipid bilayer influence the function of membrane-bound proteins (79). Given the importance of mechanics in outer hair cell function, quantifying the effect of salicylate on bilayer elastic properties is a necessary step towards understanding the mechanism of its effect on hearing. 


\section{Undulation and bending modulus}

Small molecule binding is known to influence the undulation and bending mechanics of lipid bilayers $(34,80)$. A quantitative analysis of the undulation of a lipid bilayer was performed for the six simulated systems using the spectral methods of Lindahl and Edholm (27). Membrane undulation was described via a height variable $h(x, y)$ for each lipid defined by the position of the first phospholipid glycol carbon (i.e., the carbon connecting the tails to the head group).

The choice of the glycol carbon for the height definition differs from the volume calculations carried out above (using the phosphorous); this choice was made for the purposes of comparison with the results of Lindahl and Edholm (27). Height variables for each leaflet of the bilayer were mapped to two-dimensional grids with $5 \AA$ spacing in each direction using 2 ps frequency snapshots from the MD trajectory. A two-dimensional Fourier transform of the height grids was calculated according to:

$$
u(m, n)=\frac{\Delta x \Delta y}{L_{x} L_{y}} \sum_{x=0}^{L_{X}} \sum_{y=0}^{L_{Y}} h(x, y) e^{-2 \pi i\left(\frac{m x}{L_{x}}+\frac{n y}{L_{y}}\right)}
$$

where $m=0, \ldots, M, n=0, \ldots, N$, and the wave number limits are related to the Nyquist frequency, $\quad M, N=\frac{1}{2 f_{\text {Nyquist. }}}$ The undulation mode spectrum $u(q)$ was calculated by averaging over the two leaflets and reducing the two-dimensional transformed height function to a onedimensional spectrum over the magnitude of the wave vector:

$$
q=|q|=2 \pi \sqrt{\left(\frac{m}{L_{x}}\right)^{2}+\left(\frac{n}{L_{y}}\right)^{2}} .
$$

Since the area of the simulated lipid bilayer in this study is significantly larger than the membrane thickness, the membrane mechanics can be modeled by an elastic sheet via the relationship (27):

$$
\left\langle u^{2}(q)\right\rangle=\frac{1}{\beta A_{\text {box }}} \frac{1}{K_{\text {bend }} q^{4}+\gamma q^{2}}
$$

where $\beta=\left(k_{B} T\right)^{-1}$ is the inverse thermal energy, $A_{\mathrm{box}}$ is the projected area of the periodic box, $K_{\text {bend }}$ is bending modulus, $\gamma$ is the surface tension, $q$ is the wave number, and $u$ is the $q$-space mode magnitude. Since the MD simulations in this study were performed under constant pressure conditions, the surface tension was taken as zero (24) and equation (7) was rewritten as

$$
\log \left\langle u^{2}(q)\right\rangle=-\log \left(\beta A_{\text {box }} K_{\text {bend }}\right)-4 \log (q)
$$

This relationship was verified by linear regression of the logarithm of spectral intensity per mode and logarithm of wave number (see Table 3). These results indicate that the undulation spectrum scales roughly as expected $u \sim q^{-4}(27,81)$ and is independent of salicylate concentration. While the overall undulation spectrum is independent of salicylate, Figure 9 shows some qualitative examples of local changes in membrane structure and curvature due to introduction of salicylate. These changes are further illustrated in the MD trajectory movies provided at http://agave.wustl.edu/membrane/DPPC-salicylate/ and in Supporting Information. Such perturbation is in qualitative agreement with experimental studies showing that the interaction of the related amphiphile aspirin with membranes induces local conformational changes $(82,83)$. 
Although the bending modulus can be determined from equation (10), we chose to calculate the bending modulus for each system from the total root mean square (RMS) amplitude of the undulation modes in the system (27)

$$
\left\langle u^{2}\right\rangle \approx \frac{A_{\text {box }}}{257 \beta K_{\text {bend }}}
$$

where $u$ is the undulation intensity in Fourier space. Bending moduli for each of the MD systems were calculated via equation (9) with means and deviations obtained using the $5 \mathrm{~ns}$ block averaging scheme described above. The pure DPPC system gave a bending modulus of $10 \pm$ $3 \times 10^{-20} \mathrm{~J}$, a value in reasonable agreement with the experimental result of $5.5 \times 10^{-20} \mathrm{~J}$ (84). The results of these analyses are shown in Figure 10(a) and indicate that salicylate decreases the bending modulus of the bilayer in a small, statistically-insignificant $(\mathrm{p}<0.05)$ manner. These results are different than the experimental results of Zhou and Raphael (16) who showed that application of salicylate resulted in a significant decrease in the bending modulus of stearoyl-oleoylphosphatidylcholine (SOPC) giant unilamellar vesicles (GUVs) under tension. While the simulations described here could contain artifacts from finite domain sizes and finite sampling, differences with the Zhou and Raphael experiments could also be due to different lipid types (SOPC) and different surface tensions. Interestingly, our results do agree with several experiments on larger-scale systems which show no effect of salicylate on mechanical properties. In particular, membrane tether experiments (85) demonstrated that salicylate does not significantly affect the membrane mechanics of OHC lateral walls (13, $14,86)$ or HEK cell membranes $(14,86)$. Our observations are also in agreement with atomic force microscopy experiments of Zhang et al which observed salicylate-induced change in HEK membrane surface charge but no change in membrane mechanics (force-displacement profiles) (15). Similarly, the work of Hallworth (8) showed that salicylate changes the force generation of OHCs without a change in OHC stiffness. Finally, Morimoto et al demonstrated that salicylate did not change the pressure required for vesiculation of OHC lateral walls (9). Unlike the SOPC work of Zhou and Raphael, these are experiments on cells and therefore differ in contain a number of additional components (cytoskeleton, membrane proteins, etc.) not included in the simulations presented here; however, they do provide qualitative support for our observations.

\section{Area compressibility modulus}

Area compressibility moduli describe the energetics of increases and decreases in membrane areas. These moduli have previously been calculated from either surface tensions (24), fluctuations in projected membrane area $(24,34)$, or fluctuations in area per lipid (24). Since the MD simulations in this study were performed under isotropic constant pressure, fluctuations in membrane area were used to calculate the area compressibility moduli. However, to account for the contribution of bound ions and salicylate to the membrane area, we employed the total membrane solvent accessible surface area (SASA) rather than the total projected membrane area used in other MD analyses $(24,34)$. The SASA was calculated using a water-like spherical probe of $1.4 \AA$ with the GROMACS 3.2.1 software. We then determined the area compressibility moduli of the simulated systems from the fluctuations in the SASA according to the usual formula (24)

$$
K_{\text {area }}=\frac{A}{\beta \sigma_{A}^{2}}
$$

where $K_{\text {area }}$ is the area compressibility modulus, $A$ is the SASA, and $\sigma_{\mathrm{A}}$ is the fluctuation of the SASA. Means and averages for the moduli were determined from the trajectory using the block averaging scheme described above. The pure DPPC simulation gave a compressibility 
modulus of $70 \pm 20 \mathrm{mN} \mathrm{m}^{-1}$, a value which underestimates the experimental result of $250 \mathrm{mN}$ $\mathrm{m}^{-1}(64,87)$. Differences between this value and experiment could be due to a number of reasons, including finite size and sampling effects (24) and the specific pressure regulation method used for this simulation. The results for the six systems are presented in Figure 10(b) and demonstrate that salicylate did not significantly decrease the area compressibility modulus of the DPPC bilayer.

As with the bending moduli reported above, these results are different than the SOPC GUV experiments of Zhou and Raphael (16) but agree with studies of salicylate effect on cellular mechanics $(8,9,13-15,86)$.

\section{Bulk modulus}

The bulk modulus measures the volume compressibility of the system. For our constant pressure simulations, the bulk moduli were calculated from the fluctuations of the volume of the system according to (88):

$$
K_{\text {bulk }}=\frac{V}{\beta \sigma_{V}^{2}}
$$

where $K_{\text {bulk }}$ is the bulk modulus, $V$ is the volume of the lipid bilayer, and $\sigma_{\mathrm{V}}$ is the fluctuation of the volume of the lipid bilayer. The volume of the lipid bilayer was calculated using the lipid phosphorous position in the same manner as the per-lipid volume calculations described above. Bulk moduli means and deviations were determined by the $5 \mathrm{~ns}$ block averaging method described above. The lipid bilayer bulk moduli for the 6 simulated systems are plotted in Figure 10 (c); the results show that salicylate did not significantly affect the bulk modulus.

Bulk moduli were not available from the SOPC GUV experiments of Zhou and Raphael (16); however, our results do agree with studies of salicylate effects on cell mechanics $(8,9,13-15$, 86). However, our calculated bulk modulus for the pure DPPC lipid bilayer at a temperature of $323 \mathrm{~K}$ was $(6.1 \pm 0.3) \times 10^{8} \mathrm{~N} \mathrm{~m}^{-2}$. Like the area compressibility modulus, this value differs from the experimental measurements of $2.2 \times 10^{9} \mathrm{~N} \mathrm{~m}^{-2}$ for DPPC (89). Possible reasons for this difference were discussed above and include the usual potential finite size and sampling artifacts associated with MD simulations (24).

\section{Conclusions}

The effect of salicylate on the microscopic and mesoscopic properties of a DPPC lipid bilayer was investigated with molecular dynamics simulations. Results from this study showed that salicylate significantly affected a number of bilayer structural properties including a reduction in the membrane area and increased ordering of the lipid acyl tails. These changes were interpreted in terms of the tight coordination of salicylate by the DPPC head groups and the nearly perpendicular orientation of salicylate in the bilayer which permitted tight membrane packing, a mechanism which is supported by studies of salicylate-induced structural changes in micelles (69). Salicylate introduced a local increase in $\mathrm{Na}^{+}$and a decrease in $\mathrm{Cl}^{-}$near the bilayer-water interface. This competition between salicylate and chloride was qualitatively supported by similar observations in OHCs (21). The binding of this amphiphile also changed the electrostatic potential and dielectric response of the system, with significant changes near the membrane-water interface. These results were shown to be in qualitative agreement with a number of experimental observations, including salicylate-induced changes in membrane electrostatic potentials $(17,72)$ and surface charges $(15)$. Finally, we observed no changes in the mechanical properties of the system due to salicylate binding. This lack of change was discussed in light of experimental evidence (on somewhat different membrane systems) which supported $(8,9,13,15,86)$ and contradicted (16) our observations. Interestingly, most of the salicylate effects described above were independent of dosage; an observation which is in 
reasonable agreement with the results of Kakehata et al who found that $\mathrm{OHC}$ response to salicylate was saturated at $\sim 10 \mathrm{mM}$ (19).

As mentioned in the introduction, the ultimate goal of this research was to help understand the hearing-related side effects of salicylate in the context of OHC membrane electrical and mechanical properties. There is clearly a significant gap between the existing DPPC model bilayer and the complexity of the $\mathrm{OHC}$ and its biological surroundings. Fortunately, there are a number of recent computational developments, including methods to simulate asymmetric electrolyte solutions and potential gradients around lipid bilayers (90) and coarse-grained simulations of large biomembranes $(28,29)$, which will allow us to pursue more realistic models of amphiphile effects on $\mathrm{OHC}$ hearing in the future. Therefore, present models provide important insight into salicylate interaction with model membranes and the basis for future multiscale research to understand the macroscopic effects of ligand-induced changes in membrane properties.

\section{Acknowledgements}

YS would like to thank M. Bradley and T. Dolinsky for helpful discussions. NAB and YS thank Dr. W. E. Brownell for the initial suggestion of this project and continuing insightful discussions, Drs. B. Farrell and R. M. Raphael for numerous helpful suggestions on the project and the manuscript, M. Kaneda for her preliminary work on this project, and M. Bradley and A. Vitalis for critical reading of the manuscript. This work was supported in part by grants from the NIH and Alfred P. Sloan Foundation to NAB.

\section{References}

1. Roberts, LJ., II; Morrow, JD. Analgesic-antipyretic and antiinflammatory agents and drugs employed in the treament of gout. In: Hardman, JG.; Limbird, LE.; Goodman Gilman, A., editors. The Pharmacological Basis of Therapeutics. McGraw-Hill Medical Publishing Division; Chicago, IL: 2001.

2. Shehata WE, Brownell WE, Dieler R. Effects of salicylate on shape, electromotility and membrane characteristics of isolated outer hair cells from guinea pig cochlea. Acta Oto-laryngologica 1991;111:707-18. [PubMed: 1950533]

3. Myers EN, Bernstein JM. Salicylate ototoxicity; a clinical and experimental study. Archives of Otolaryngology 1965;82:483-93.

4. Myers EN, Bernstein JM, Fostiropolous G. Salicylate ototoxicity: a clinical study. New England Journal of Medicine 1965;273:587-90. [PubMed: 14329630]

5. Jung TT, Rhee CK, Lee CS, Park YS, Choi DC. Ototoxicity of salicylate, nonsteroidal antiinflammatory drugs, and quinine. Otolaryngologic clinics of North America 1993;26:791-810. [PubMed: 8233489]

6. Tunstall MJ, Gale JE, Ashmore JF. Action of salicylate on membrane capacitance of outer hair cells from the guinea-pig cochlea. Journal of Physiology 1995;485:739-52. [PubMed: 7562613]

7. Lue AJ, Brownell WE. Salicylate induced changes in outer hair cell lateral wall stiffness. Hearing Research 1999;135:163-8. [PubMed: 10491964]

8. Hallworth R. Modulation of outer hair cell compliance and force by agents that affect hearing. Hearing Research 1997;114:204-12. [PubMed: 9447933]

9. Morimoto N, Raphael RM, Nygren A, Brownell WE. Excess plasma membrane and effects of ionic amphipaths on mechanics of outer hair cell lateral wall. American Journal of Physiology -- Cell Physiology 2002;282:C1076-86. [PubMed: 11940523]

10. Stewart CE, Hudspeth AJ. Effects of salicylates and aminoglycosides on spontaneous otoacoustic emissions in the Tokay gecko. Proceedings of the National Academy of Sciences of the United States of America 2000;97:454-9. [PubMed: 10618439]

11. Grosh K, Zheng J, Zou Y, de Boer E, Nuttall AL. High-frequency electromotile responses in the cochlea. Journal of the Acoustical Society of America 2004;115:2178-84. [PubMed: 15139629]

12. Ashmore J, Gale J. The cochlear amplifier. Current Biology 2004;14:R403-R404. [PubMed: 15182680] 
13. Ermilov, S.; Brownell, WE.; Anvari, B. Proceedings of SPIE. Cartwright, AN., editor. SPIE -- The International Society for Optical Engineering; 2004. p. 136-42.

14. Anvari, B.; Qian, F.; Pereria, FA.; Brownell, WE. Prestin-lacking membranes are capable of high frequency electro-mechanical transduction. In: Nuttall, AL., editor. Auditory Mechanisms: Processes and Models. World Scientific; London: 2005. In press

15. Zhang PC, Keleshian AM, Sachs F. Voltage-induced membrane movement. Nature 2001;413:42832. [PubMed: 11574890]

16. Zhou Y, Raphael RM. Effect of salicylate on the elasticity, bending stiffness, and strength of SOPC membranes. Biophysical Journal. 2004in press

17. Farrell B, Shope CD, Brownell WE. Voltage Dependent Capacitance of Human Embryonic Kidney (HEK) Cells. 2005in review

18. Mazorow DL, Haug A, Bull R, McGroarty EJ. Effects of aspirin, indomethacin, and sodium salicylate on human erythrocyte membranes as detected with electron spin resonance spectroscopy. Thrombosis Research 1985;40:779-92. [PubMed: 3003962]

19. Kakehata S, Santos-Sacchi J. Effects of salicylate and lanthanides on outer hair cell motility and associated gating charge. Journal of Neuroscience 1996;16:4881-9. [PubMed: 8756420]

20. Oghalai JS, Zhao HB, Kutz JW, Brownell WE. Voltage- and Tension-Dependent Lipid Mobility in the Outer Hair Cell Plasma Membrane. Science 2000;287:658-661. [PubMed: 10650000]

21. Oliver D, He DZ, Klocker N, Ludwig J, Schulte U, Waldegger S, Ruppersberg JP, Dallos P, Fakler B. Intracellular anions as the voltage sensor of prestin, the outer hair cell motor protein. Science 2001;292:2340-3. [PubMed: 11423665]

22. Dong X-X, Iwasa KH. Tension Sensitivity of Prestin: Comparison with the Membrane Motor in Outer Hair Cells. Biophysical Journal 2004;86

23. Song L, Seeger A, Santos-Sacchi J. On Membrane Motor Activity and Chloride Flux in the Outer Hair Cell: Lessons Learned from the Environmental Toxin Tributyltin. Biophysical Journal 2005;88:2350-62. [PubMed: 15596517]

24. Feller SE, Pastor RW. Constant surface tension simulations of lipid bilayers: The sensitivity of surface areas and compressibilities. Journal of Chemical Physics 1999;111:1281-7.

25. Pandit SA, Bostick D, Berkowitz ML. Mixed Bilayer Containing Dipalmitoylphosphatidylcholine and Dipalmitoylphosphatidylserine: Lipid Complexation, Ion Binding, and Electrostatics. Biophysical Journal 2003;85:3120-3131. [PubMed: 14581212]

26. Tieleman DP, Berendsen HJC. Molecular dynamics simulations of a fully hydrated dipalmitoylphosphatidylcholine bilayer with different macroscopic boundary conditions and parameters. Journal of Chemical Physics 1996;105:4871-80.

27. Lindahl E, Edholm O. Mesoscopic undulations and thickness fluctuations in lipid bilayers. Biophysical Journal 2000;79:426-433. [PubMed: 10866968]

28. Ayton G, Voth GA. Bridging microscopic and mesoscopic simulations of lipid bilayers. Biophysical Journal 2002;83:3357-70. [PubMed: 12496103]

29. Pickholz M, Saiz L, Klein ML. Concentration Effects of Volatile Anesthetics on the Properties of Model Membranes: A Coarse-Grain Approach. Biophysical Journal 2005;88:1524-34. [PubMed: 15613628]

30. Sachs JN, Woolf TB. Understanding the Hofmeister effect in interactions between chaotropic anions and lipid bilayers: molecular dynamics simulations. Journal of the American Chemical Society 2003;125:8742-8743. [PubMed: 12862466]

31. Pasenkiewicz-Gierula M, Róg T, Grochowski J, Serda P, Czarnecki R, Librowski R, Lochyski S. Effects of a Carane Derivative Local Anesthetic on a Phospholipid Bilayer Studied by Molecular Dynamics Simulation. Biophysical Journal 2003;85:1248-1258. [PubMed: 12885668]

32. Pereira CS, Lins RD, Chandrasekhar I, Freitas LCG, Hünenberger PH. Interaction of the Disaccharide Trehalose with a Phospholipid Bilayer: A Molecular Dynamics Study. Biophysical Journal 2004;86:2273-2285. [PubMed: 15041666]

33. Smondyrev AM, Berkowitz ML. Molecular dynamics simulation of DPPC bilayer in DMSO. Biophysical Journal 1999;76:2472-2478. [PubMed: 10233064]

34. Hofsäß C, Lindahl E, Edholm O. Molecular Dynamics Simulations of Phospholipid Bilayers with Cholesterol. Biophysical Journal 2003;84:2192-206. [PubMed: 12668428] 
35. Villareal MA, Díaz SB, Disalvo EA, Montich GG. Molecular Dynamics Simulation Study of the Interaction of Trehalose with Lipid Membranes. Langmuir 2000;20:7844-51.

36. Lee BW, Faller R, Sum AK, Vattulainen I, Patra M, Karttunen M. Structural effects of small molecules on phospholipid bilayers investigated by molecular simulations. Fluid Phase Equilibria 2004;225:6368.

37. Sum AK, Faller R, de Pablo JJ. Molecular Simulation Study of Phospholipid Bilayers and Insights of the Interactions with Disaccharides. Biophysical Journal 2003;85:2830-44. [PubMed: 14581188]

38. Dickey AN, Faller R. Investigating interactions of biomembranes and alcohols: A multiscale approach. Journal of Polymer Science Part B: Polymer Physics 2005;43:1025-32.

39. Murray D, Honig B. Electrostatic control of the membrane targeting of C2 domains. Molecular Cell 2002;9:145-154. [PubMed: 11804593]

40. Diraviyam K, Stahelin RV, Cho W, Murray D. Computer modeling of the membrane interaction of FYVE domains. Journal of Molecular Biology 2003;328:721-36. [PubMed: 12706728]

41. Lopez CF, Nielsen SO, Moore PB, Klein ML. Understanding nature's design for a nanosyringe. Proceedings of the National Academy of Sciences of the United States of America 2004;101:44314434. [PubMed: 15070735]

42. Zhu F, Tajkhorshid E, Schulten K. Theory and Simulation of Water Permeation in Aquaporin-1. Biophysical Journal 2004;86:50-57. [PubMed: 14695248]

43. Lindahl E, Hess B, van der Spoel D. GROMACS 3.0: a package for molecular simulation and trajectory analysis. Journal of Molecular Modeling 2001;7:306-317.

44. Kleywegt GJ, Jones TA. Databases in protein crystallography. Acta Crystallographica D 1998;54:1119-31.

45. van Aalten DMF, Bywater R, Findlay JBC, Hendlich M, Hooft RWW, Vriend G. PRODRG, a program for generating molecular topologies and unique molecular descriptors from coordinates of small molecules. Journal of Computer-Aided Molecular Design 1996;10:255-262. [PubMed: 8808741]

46. Schrödinger, I. (2001), Schrödinger, Inc.

47. Philipp DM, Friesner RA. Mixed ab initio QM/MM modeling using frozen orbitals and tests with alanine dipeptide and tetrapeptide. Journal of Computational Chemistry 1999;20:1468-94.

48. Murphy RB, Philipp DM, Friesner RA. A mixed quantum mechanics/molecular mechanics (QM/ $\mathrm{MM}$ ) method for large-scale modeling of chemistry in protein environments. Journal of Computational Chemistry 2000;21:1442-57.

49. Kaminski G, Friesner RA, Tirado-Rives J, Jorgensen WL. Evaluation and reparameterization of the OPLS-AA force field for proteins via comparison with accurate quantum chemical calculations on peptides. Journal of Physical Chemistry B 2001;105:6474-6487.

50. Ponder JW, Case DA. Force fields for protein simulations. Advances in Protein Chemistry 2003;66:27-85. [PubMed: 14631816]

51. Berger O, Edholm O, Jahnig F. Molecular dynamics simulations of a fluid bilayer of dipalmitoylphosphatidylcholine at full hydration, constant pressure, and constant temperature. Biophysical Journal 1997;72:2002-13. [PubMed: 9129804]

52. Nagle JF, Wiener MC. Structure of fully hydrated bilayer dispersions. Biochimica et Biophysica Acta 1988;942:1-10. [PubMed: 3382651]

53. Seelig A, Seelig J. Dynamic structure of fatty acyl chains in a phospholipid bilayer measured by deuterium magnetic resonance. Biochemistry 1974;13:4839-45. [PubMed: 4371820]

54. Marrink SJ, Berger O, Tieleman P, Jähnig F. Adhesion Forces of Lipids in a Phospholipid Membrane Studied by Molecular Dynamics Simulations. Biophysical Journal 1998;74:931-43. [PubMed: 9533704]

55. Berendsen HJC, Grigera JR, Straatsma TP. The missing term in effective pair potentials. Journal of Physical Chemistry 1987;91:6269-71.

56. Böckmann RA, Hac A, Heimburg T, Grubmüller H. Effect of Sodium Chloride on a Lipid Bilayer. Biophysical Journal 2003;85:1647-55. [PubMed: 12944279] 
57. Nagle JF, Zhang R, Tristram-Nagle S, Sun W, Petrache HI, Suter RM. X-ray structure determination of fully hydrated L alpha phase dipalmitoylphosphatidylcholine bilayers. Biophysical Journal 1996;70:1419-31. [PubMed: 8785298]

58. Parrinello M, Rahman A. Polymorphic transitions in single crystals: A new molecular dynamics method. Journal of Applied Physics 1981;52:7182-90.

59. Hoover WG. Canonical dynamics: Equilibrium phase-space distributions. Physical Review A 1985;31:1695-97. [PubMed: 9895674]

60. Darden T, York D, Pedersen LG. Particle mesh Ewald: An N·log(N) method for Ewald sums in large systems. Journal of Chemical Physics 1993;98:10089-92.

61. Patra M, Karttunen M, Hyvönen MT, Falck E, Vattulainen I. Lipid Bilayers Driven to a Wrong Lane in Molecular Dynamics Simulations by Subtle Changes in Long-Range Electrostatic Interactions. Journal of Physical Chemistry B 2004;108:4485-4494.

62. Patra M, Karttunen M, Hyvönen MT, Falck E, Lindqvist P, Vattulainen I. Molecular Dynamics Simulations of Lipid Bilayers: Major Artifacts Due to Truncating Electrostatic Interactions. Biophysical Journal 2003;84:3636-3645. [PubMed: 12770872]

63. Petrache HI, Dodd SW, Brown MF. Area per Lipid and Acyl Length Distributions in Fluid Phosphatidylcholines Determined by 2H NMR Spectroscopy. Biophysical Journal 2000;79:317292. [PubMed: 11106622]

64. Nagle JF, Tristram-Nagle S. Structure of lipid bilayers. Biochimica et Biophysica Acta (BBA) Reviews on Biomembranes 2000;1469:159-95.

65. Egberts E, Berendsen HJC. Molecular dynamics simulation of a smectic liquid crystal with atomic detail. Journal of Chemical Physics 1988;89:3718-32.

66. Bailey, NTJ. Statistical Methods in Biology. 3. Cambridge University Press; New York: 1995.

67. Flyvbjerg H, Petersen HG. Error estimates on averages of correlated data. Journal of Chemical Physics 1989;91:461-6.

68. Koubi L, Tarek M, Klein ML, Scharf D. Distribution of Halothane in a Dipalmitoylphosphatidylcholine Bilayer from Molecular Dynamics Calculations. Biophysical Journal 2000;78:800-11. [PubMed: 10653792]

69. Lin Z, Cai JJ, Scriven LE, Davis HT. Spherical-to-Wormlike Micelle Transition in CTAB Solutions. Journal of Physical Chemistry 1994;98:5984-93.

70. Sachs JN, Nanda H, Petrache HI, Woolf TB. Changes in Phosphatidylcholine Headgroup Tilt and Water Order Induced by Monovalent Salts: Molecular Dynamics Simulations. Biophysical Journal 2004;86:3772-82. [PubMed: 15189873]

71. Nwafor A, Coakley WT. Drug-induced shape change in erythrocytes correlates with membrane potential change and is independent of glycocalyx charge. Biochemical Pharmacology 1985;34:3329-36. [PubMed: 4038341]

72. McLaughlin S. Salicylates and phospholipid bilayer membranes. Nature 1973;243:234-235. [PubMed: 4706296]

73. Cohen I, Noble D, Ohba M, Ojeda C. Action of salicylate ions on the electrical properties of sheep cardiac Purkinje fibres. Journal of Physiology 1979;297:163-85. [PubMed: 536909]

74. Levitan H, Barker JL. Membrane Permeability: Cation Selectivity Reversibly Altered by Salicylate. Science 1972;178:63-4. [PubMed: 5070517]

75. Levitan H, Barker JL. Salicylate: A Structure-Activity Study of its Effects on Membrane Permeability. Science 1972;176:1423-5. [PubMed: 5033649]

76. Attwell D, Bergman C, Ojeda C. The action of salicylate ions on the frog node of Ranvier. Journal of Physiology 1979;295:69-81. [PubMed: 316452]

77. Ballenegger V, Hansen JP. Dielectric permittivity profiles of confined polar fluids. Journal of Chemical Physics 2005;122:114711. [PubMed: 15836247]

78. Lin JH, Baker NA, McCammon JA. Bridging the implicit and explicit solvent approaches for membrane electrostatics. Biophysical Journal 2002;83:1374-1379. [PubMed: 12202363]

79. Roux B, Schulten K. Computational Studies of Membrane Channels. Structure 2004;12:1343-51. [PubMed: 15296727] 
80. Ly HV, Longo ML. The Influence of Short-Chain Alcohols on Interfacial Tension, Mechanical Properties, Area/Molecule, and Permeability of Fluid Lipid Bilayers. Biophysical Journal 2004;87:1013-33. [PubMed: 15298907]

81. Goetz R, Gompper G, Lipowsky R. Mobility and Elasticity of Self-Assembled Membranes. Physical Review Letters 1999;82:221-4.

82. Watala C, Gwozdzinski K. Effect of aspirin on conformation and dynamics of membrane proteins in platelets and erythrocytes. Biochemical Pharmacology 1993;45:1343-9. [PubMed: 8385461]

83. Sheetz MP, Singer SJ. Equilibrium and kinetic effects of drugs on the shapes of human erythrocytes. Journal of Cell Biology 1976;70:247-51. [PubMed: 932100]

84. Petrache HI, Gouliaev N, Tristram-Nagle S, Zhang R, Suter RM, Nagle JF. Interbilayer interactions from high-resolution x-ray scattering. Physical Review E 1998;57:7014-24.

85. Qian F, Ermilov S, Murdock D, Brownell WE, Anvari B. Combining optical tweezers and patch clamp for studies of cell membrane electromechanics. Review of Scientific Instruments 2004;75:2937-42.

86. Ermilov SA, Murdock DR, Brownell WE, Anvari B. Effects of salicylate on plasma membrane mechanics. Journal of Neurophysiology. 2005in press

87. Rawicz W, Olbrich KC, McIntosh T, Needham D, Evans E. Effect of Chain Length and Unsaturation on Elasticity of Lipid Bilayers. Biophysical Journal 2000;79:328-39. [PubMed: 10866959]

88. Hansen, J-P.; McDonald, IR. Theory of Simple Liquids. 2. Academic Press; San Diego, CA: 2000.

89. Mitaku S, Ikegami A, Sakanishi A. Ultrasonic studies of lipid bilayer. Phase transition in synthetic phosphatidylcholine liposomes. Biophysical Chemistry 1978;8:295-304. [PubMed: 581558]

90. Sachs JN, Crozier PS, Woolf TB. Atomistic simulations of biologically realistic transmembrane potential gradients. Journal of Chemical Physics 2004;121:10847-51. [PubMed: 15634036] 


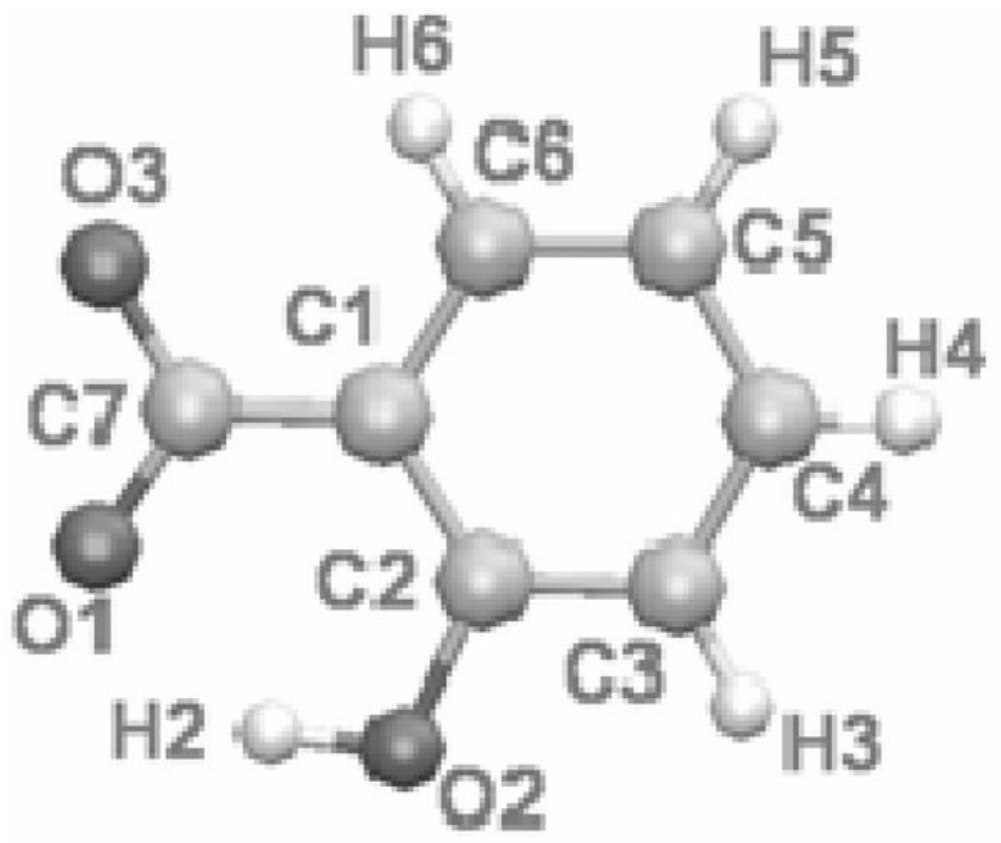

Figure 1.

Salicylate, including the atom naming scheme used elsewhere in this manuscript. 
(a)

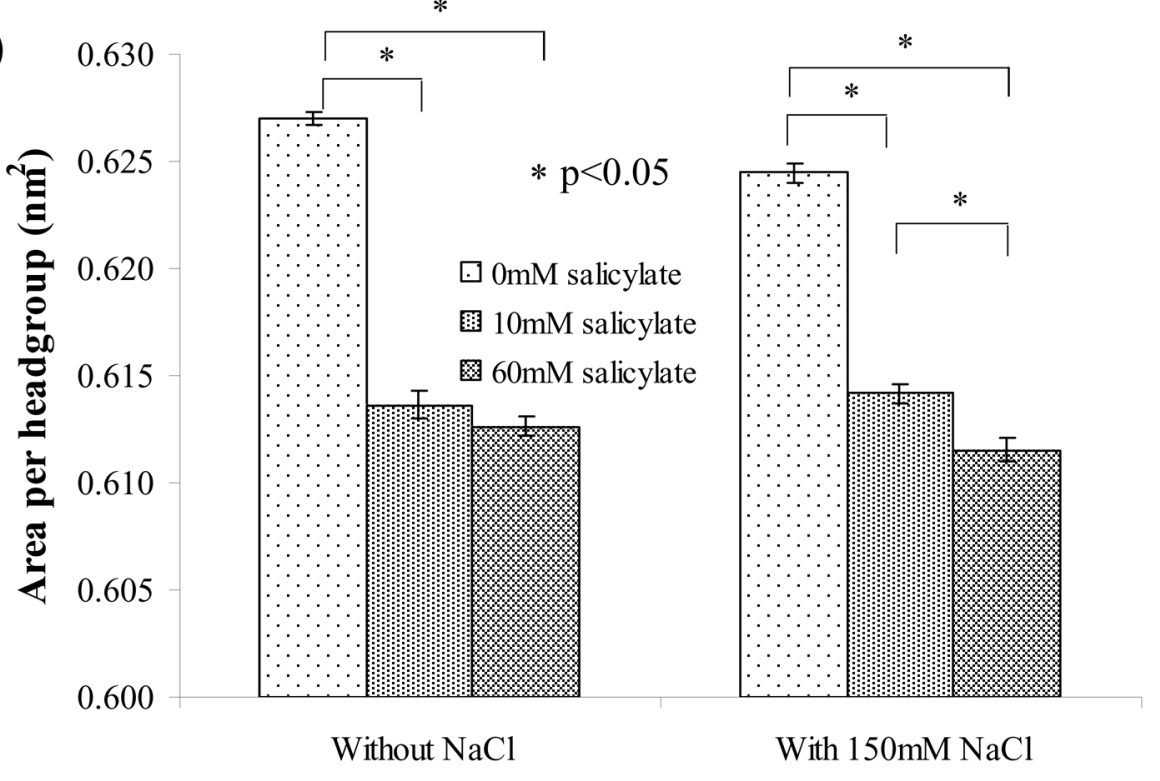

Cases (b)

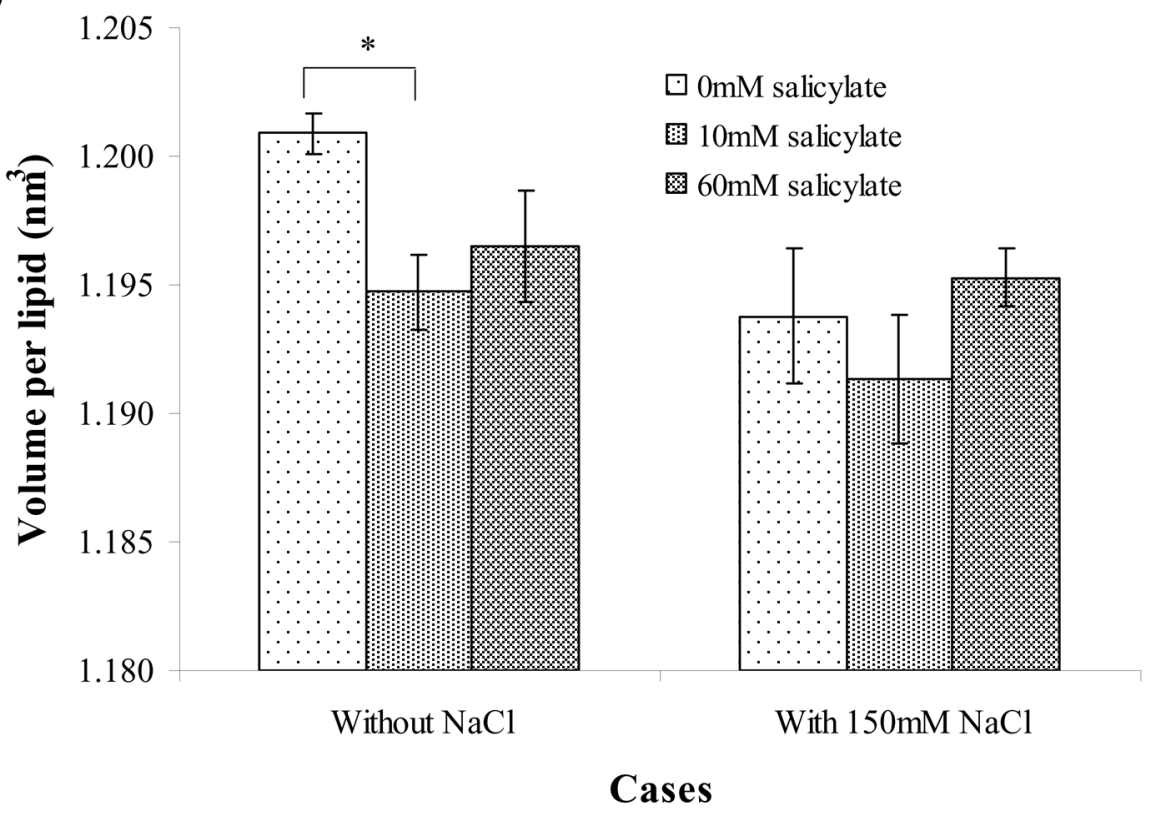

Figure 2.

The effect of salicylate on (a) lipid head group areas and (b) per-lipid volumes. Error bars were calculated as described in the text. 

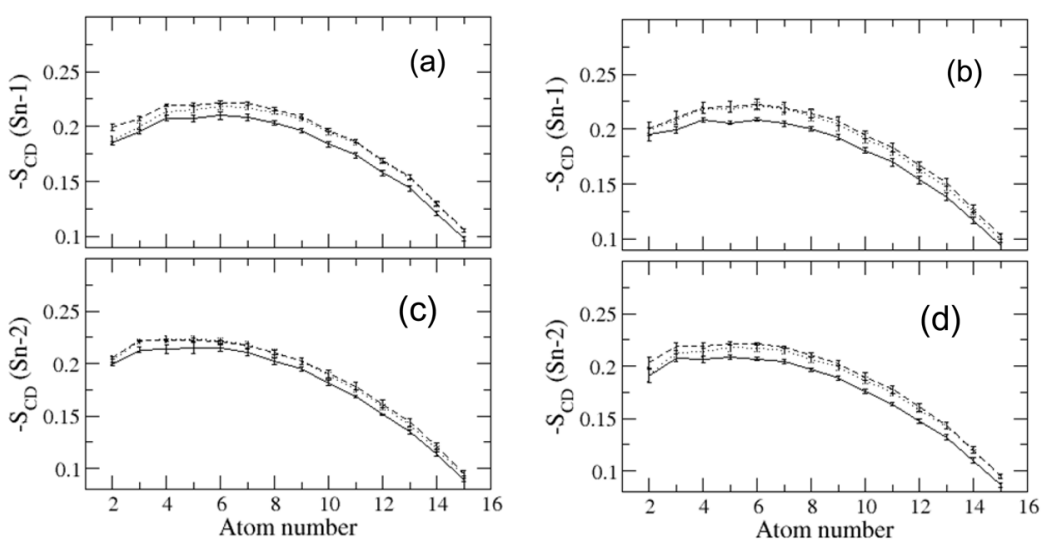

Figure 3.

Salicylate effects on the order parameters of DPPC acyl chains for (a) $n-1$ order parameters without $\mathrm{NaCl}$, (b) $n-1$ order parameters with $150 \mathrm{mM} \mathrm{NaCl}$, (c) $n-2$ order parameters without $\mathrm{NaCl}$, (d) $n-2$ order parameters with $150 \mathrm{mM} \mathrm{NaCl}$. The different lines denote $0 \mathrm{mM} \mathrm{SAL}$ (solid line), $10 \mathrm{mM}$ SAL (dotted line), and 60mM SAL (dashed line). Error bars were calculated as described in the text. 

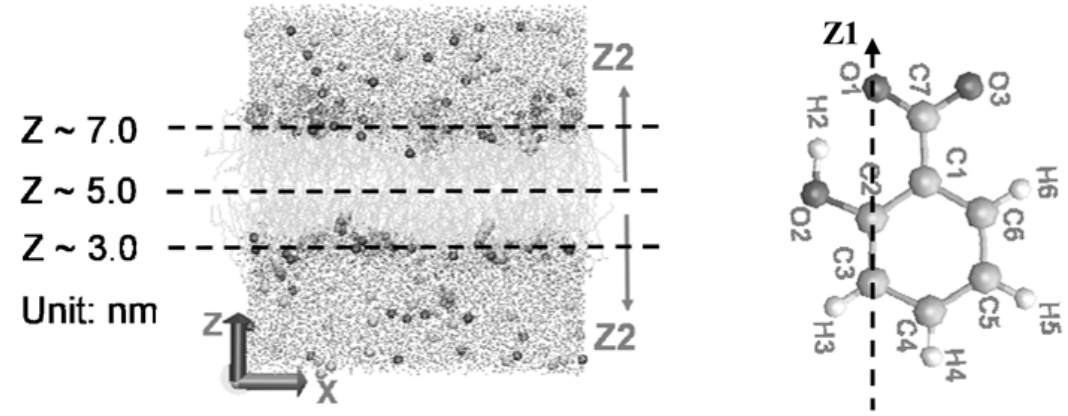

(a)

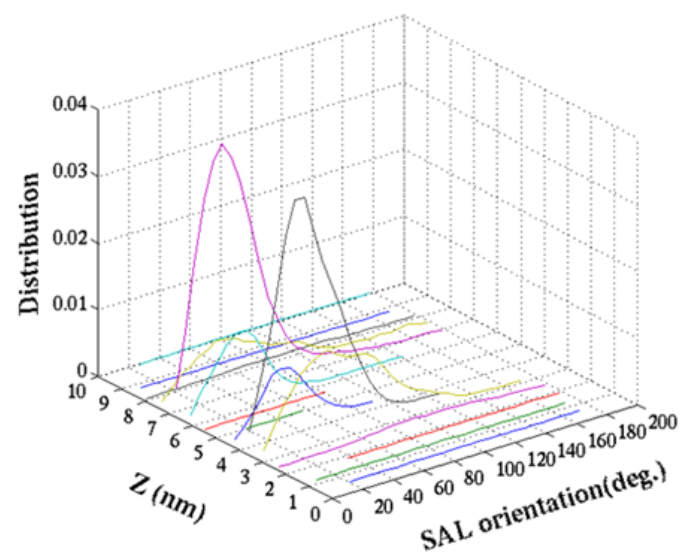

(b)

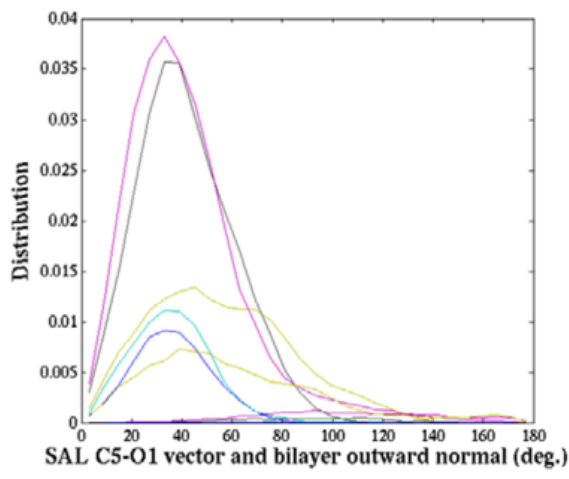

(c)

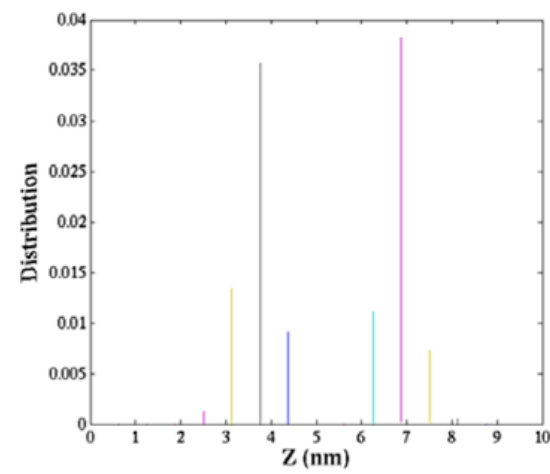

(d)

Figure 4.

Salicylate (SAL) orientation \& position distribution within the simulated system (DPPC/ $60 \mathrm{mMSAL} / \mathrm{NaCl}$ system). (a) Salicylate orientation is defined as the angle between $\mathrm{C} 3-\mathrm{O} 1$ connection of salicylate (Z1 axis) and bilayer outward normal (Z2 axis); (b) 3-D representation of salicylate orientation and position distribution; (c) 2-D representation of salicylate orientation distribution; (d) 2-D representation of salicylate position distribution. 

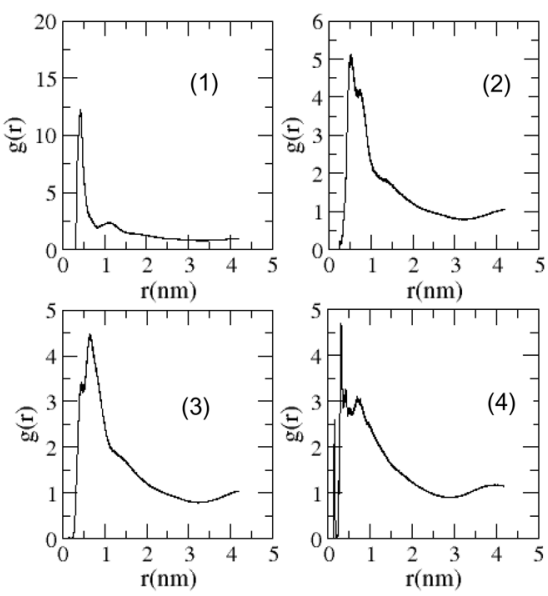

(4)

(a)

Figure 5.

Coordination of salicylate by the DPPC lipids for the DPPC/60mMSAL/NaCl system (plots for the other systems are provided in Supporting Information). (a) Radial distribution functions with respect to the salicylate hydrophilic group for various DPPC components including choline nitrogen (1), phosphate phosphorus (2), phosphate oxygen (3), and carbonyl-oxygen (4). (b) A snapshot of the MD trajectory showing the salicylate-lipid coordination. 


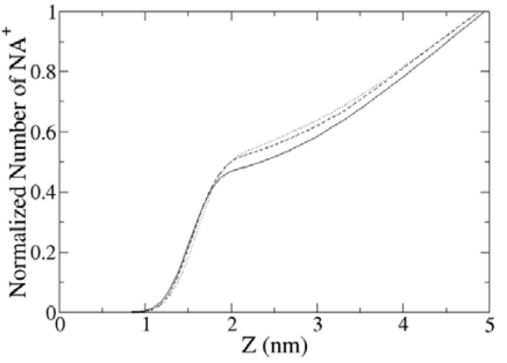

(a)

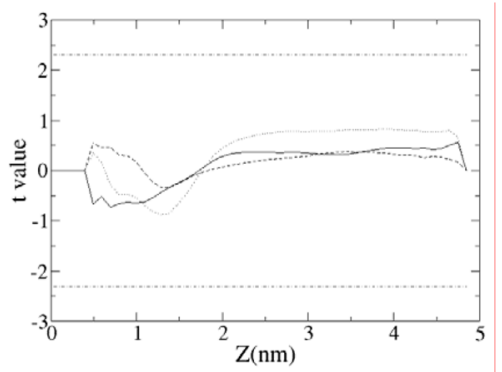

(c)

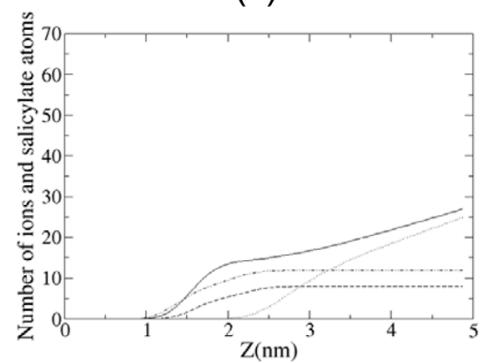

(e)

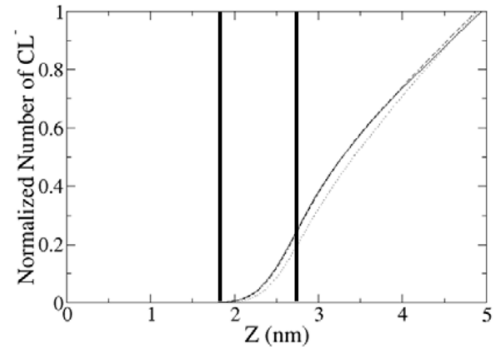

(b)

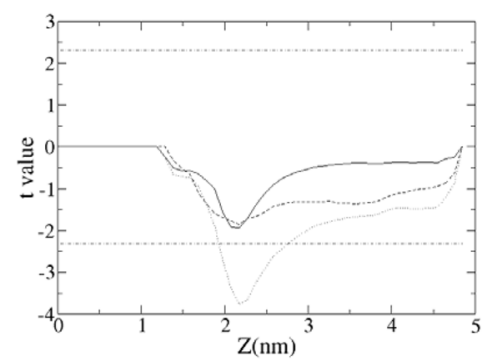

(d)

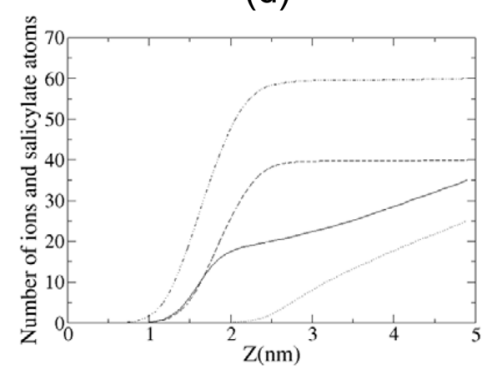

(f)

Figure 6.

Distribution of ions and salicylate atoms between the lipid bilayer center as a function of the position along the lipid bilayer normal direction. For reference, the membrane-water interface lies between $z=2-3 \mathrm{~nm}$. The top two figures show normalized cumulative number densities for (a) $\mathrm{Na}^{+}$for the three $\mathrm{NaCl}$-containing simulations, (b) $\mathrm{Cl}^{-}$for the three $\mathrm{NaCl}$-containing simulations. In (a) and (b), the lines represent different salicylate concentrations: $0 \mathrm{mM} \mathrm{SAL}$ (solid), $10 \mathrm{mM} \mathrm{SAL} \mathrm{(dashed),} \mathrm{and} 60 \mathrm{mM} \mathrm{SAL}$ (dotted). The middle figures show Student $t$ values for the number distributions of (c) $\mathrm{Na}^{+}$and (d) $\mathrm{Cl}^{-}$with for $10 \mathrm{mM} \mathrm{SAL}$ with respect to 0mM SAL (solid line), 60mM SAL with respect to 0mM SAL (dotted line), and 60mM SAL with respect to $10 \mathrm{mM}$ SAL (dashed line). $t$ values corresponding to $5 \%$ probability are shown as dotted-dashed lines. The quantities for the number densities were averaged over both leaflets of the bilayer, error bars were calculated as described in the text. Salicylate significantly decreased $(\mathrm{p}<0.05)$ the $\mathrm{Cl}^{-}$concentrations at the region between the two solid vertical bars. Finally, the bottom figures show the raw cumulative number densities for $\mathrm{Na}^{+}$(solid), $\mathrm{Cl}^{-}$ (dotted), SAL hydrophilic group (dashed), and SAL hydrophobic (dashed-dotted) from the (e) $10 \mathrm{mM}$ SAL and (f) $60 \mathrm{mM}$ SAL simulations. 


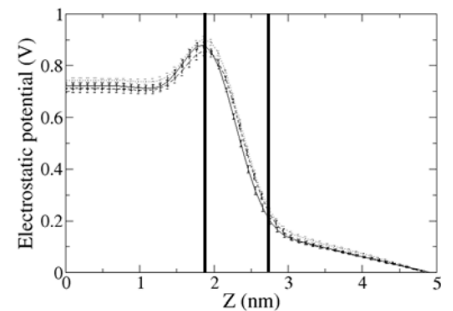

(a)

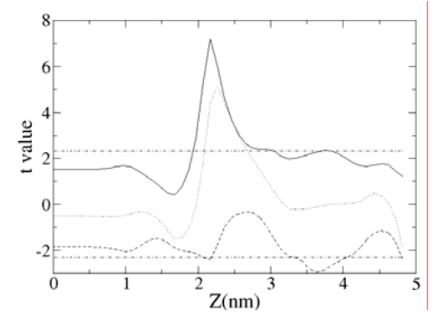

(c)

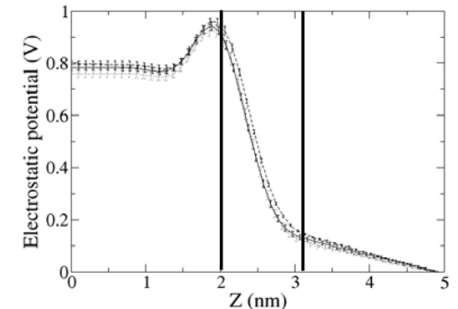

(b)

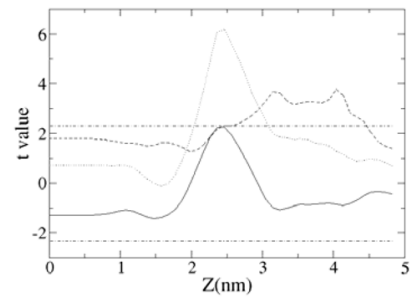

(d)

Figure 7.

Changes in the electrostatic potential due to salicylate. The top two figures show the electrostatic potential profile for (a) the simulations without added $\mathrm{NaCl}$ and (b) with $150 \mathrm{mM}$ $\mathrm{NaCl}$ for $0 \mathrm{mM}$ SAL (solid line), $10 \mathrm{mM} \mathrm{SAL} \mathrm{(dotted} \mathrm{line),} \mathrm{and} 60 \mathrm{mM}$ SAL (dashed) line. The bottom figures show Student $t$-values for the electrostatic potential of (c) the simulations without added $\mathrm{NaCl}$ and (d) with $150 \mathrm{mM} \mathrm{NaCl}$ for $10 \mathrm{mM} \mathrm{SAL}$ with respect to $0 \mathrm{mM} \mathrm{SAL}$ (solid line), 60mM SAL with respect to 0mM SAL (dotted line), and 60mM SAL with respect to $10 \mathrm{mM} \mathrm{SAL} \mathrm{(dashed} \mathrm{line).} t$ values corresponding to $5 \%$ probability are shown as dotteddashed lines. The quantities for the electrostatic potential were averaged over both leaflets of the bilayer, error bars were calculated as described in the text. Salicylate significantly increased $(p<0.05)$ electrostatic potential at the regions between the two solid vertical bars. 


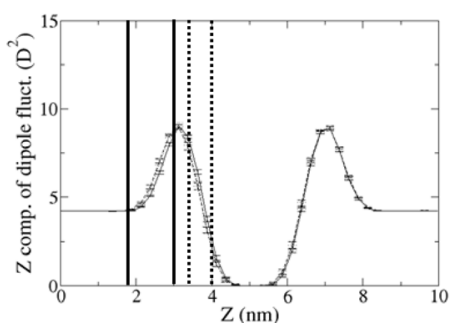

(a)

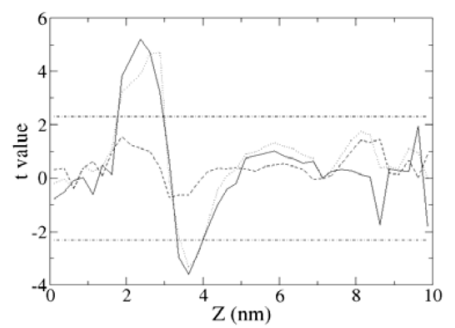

(c)

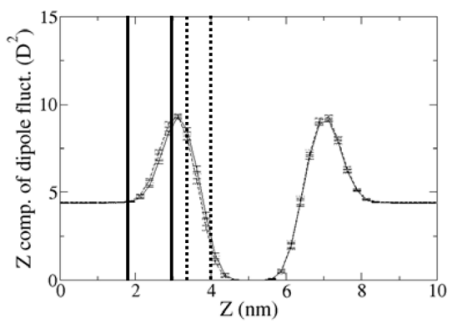

(b)

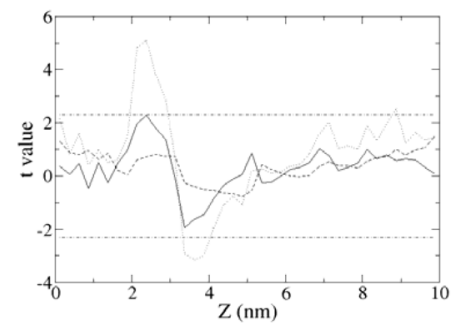

(d)

Figure 8.

Changes in the bilayer normal component of dipole moment fluctuation tensors $\left(\Gamma_{z}\right)$ for the simulated system. Since salicylate distributed unevenly across the two leaflets of the lipid bilayer during the MD simulations, the dipole moment fluctuation results were not averaged over the two leaflets. The top two plots show the dipole moment fluctuation tensor $\Gamma_{z}$ component for (a) the simulated systems without added $\mathrm{NaCl}$ and (b) the simulated systems with $150 \mathrm{mM} \mathrm{NaCl}$ for $0 \mathrm{mM} \mathrm{SAL}$ (solid line), $10 \mathrm{mM} \mathrm{SAL}$ (dotted line), and $60 \mathrm{mM} \mathrm{SAL}$ (dashed line). The bottom figures show Student $t$-values for the fluctuation tensor normal component of (c) the simulated systems without added $\mathrm{NaCl}$ and (d) the simulated systems with $150 \mathrm{mM} \mathrm{NaCl}$ for $10 \mathrm{mM}$ SAL with respect to $0 \mathrm{mM} \mathrm{SAL}$ (solid line), 60mM SAL with respect to $0 \mathrm{mM}$ SAL (dotted line), and 60mM SAL with respect to 10mM SAL (dashed line). $t$ values corresponding to $5 \%$ probability are shown as dotted-dashed lines. The error bars were calculated as described in the text. Salicylate significantly increased $(\mathrm{p}<0.05)$ dipole moment fluctuation at the regions between the two solid vertical bars and significantly decreased $(\mathrm{p}<0.05)$ dipole moment fluctuation at the regions between the two dashed vertical bars. 


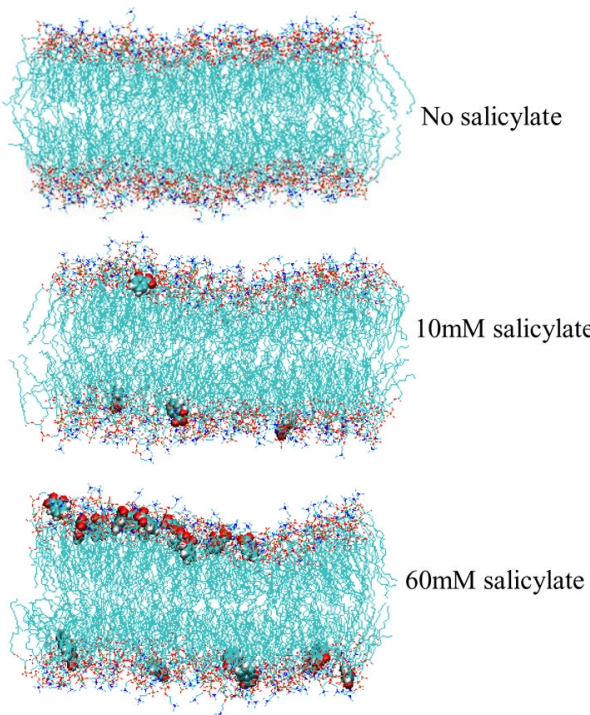

(a)

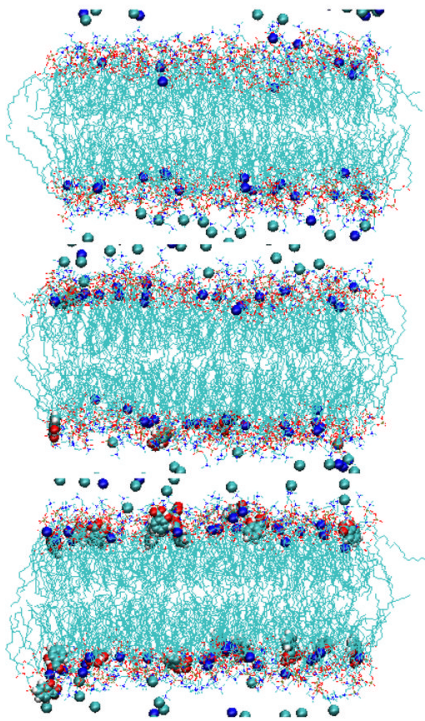

(b)

Figure 9.

Snapshots from the MD simulations (a) without $\mathrm{NaCl}$ and (b) with $150 \mathrm{mM} \mathrm{NaCl}$ for (top) systems without salicylate, (middle) systems with $10 \mathrm{mM}$ salicylate, and (bottom) systems with $60 \mathrm{mM}$ salicylate. Complete movies for these trajectories are available on the web at http://agave.wustl.edu/membrane/DPPC-salicylate/ and in Supporting Information. 


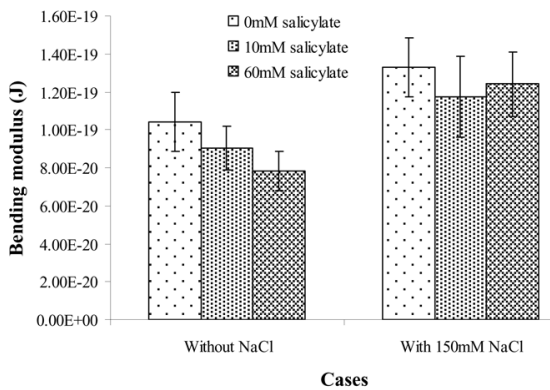

(a)

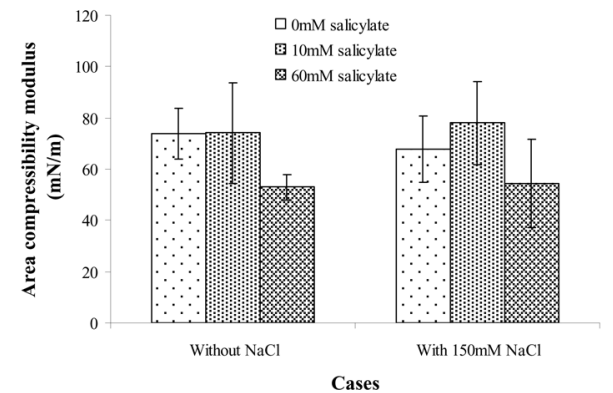

(b)

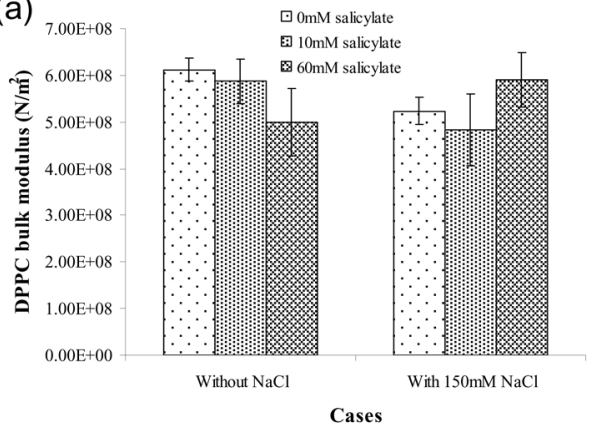

(c)

Figure 10.

Mechanical properties of the lipid bilayer with error bars were calculated as described in the text. (a) Bending modulus, (b) area compressibility modulus, and (c) bulk modulus. There were no significant differences in the simulated systems. 
Table 1

Salicylate and $\mathrm{NaCl}$ concentrations for the six systems simulated in this study.

\begin{tabular}{lll}
\hline System & Salicylate concentration(mM) & NaCl concentration (mM) \\
\hline PureDPPC & 0 & 0 \\
DPPC/10mM SAL & 10 & 0 \\
DPPC/60mM SAL & 60 & 0 \\
DPPC/NaCl & 0 & 150 \\
DPPC/10mM SAL/NaCl & 10 & 150 \\
\hline DPPC/60mM SAL/NaCl & 60 & 150 \\
\hline
\end{tabular}




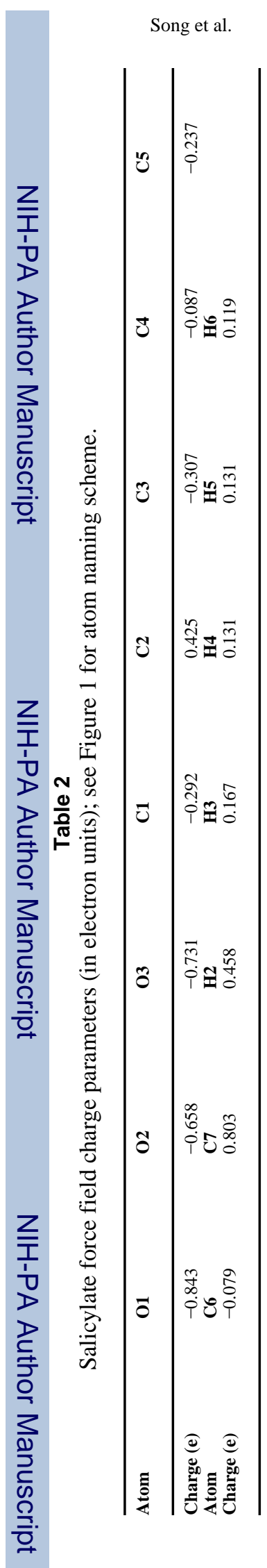

Page 27

Biochemistry. Author manuscript; available in PMC 2008 June 20. 
Table 3

The results of linear regression of the logarithm of spectral intensity per mode and the logarithm of wave number per Equation (10) and the resulting bending moduli.

\begin{tabular}{llll}
\hline Systems & Slope & Intercept & Pearson correlation coefficient \\
\hline PureDPPC & $3.47 \pm 0.05$ & $-2.76 \pm 0.03$ & -0.99 \\
DPPC/10mM SAL & $3.50 \pm 0.05$ & $-2.72 \pm 0.03$ & -0.99 \\
DPPC/60mM SAL & $3.57 \pm 0.05$ & $-2.67 \pm 0.03$ & -0.99 \\
DPPC/NaCl & $3.45 \pm 0.06$ & $-2.79 \pm 0.04$ & -0.98 \\
DPPC/10mM SAL/NaCl & $3.50 \pm 0.06$ & $-2.77 \pm 0.04$ & -0.99 \\
DPPC/60mM SAL/NaCl & $3.47 \pm 0.06$ & $-2.77 \pm 0.04$ & -0.99 \\
\hline
\end{tabular}

